\title{
Feste Kombinationen sind Mittel der Wahl in der Therapie des persistierenden Asthmas - Pro
}

\author{
R. W. Hauck
}

\author{
Fixed Combinations are the Therapy of Choice in the Treatment \\ of Persistent Asthma - Pro
}

Asthma liegt pathophysiologisch eine Kombination aus obstruktiver Ventilationsstörung und entzündlicher Veränderung der Bronchialschleimhaut zugrunde. Wenngleich $\beta$-Agonisten als die stärksten antiobstruktiv wirksamen Medikamente gewisse antiinflammatorische Eigenschaften aufweisen, so wird die antientzündliche Wirkeffektivität der Steroide nur ansatzweise erreicht. Vice versa gilt für orale wie inhalative Steroide, dass sie neben ihrem hochpotenten antientzündlichen Spektrum antiobstruktive Wirkung besitzen, die nicht mit der von $\beta$-Agonisten vergleichbar ist. Naheliegend ist somit, einer kombinierten Störung aus Obstruktion und Inflammation mit einer kombinierten Therapie aus den effektivsten antiobstruktiv und antiinflammatorischen Medikamenten entgegen zu treten. Das sind langwirkende $\beta$-Agonisten (LABA) und inhalative Steroide (ICS).

Für ihre fest kombinierte Anwendung gibt es eine Reihe von Vorteilen gegenüber den frei zu kombinierenden Präparaten. So bieten LABA und ICS pharmakodynamisch eine nahezu deckungsgleiche Wirkdauer, was ihre fest kombinierte zweimal tägliche Anwendung erlaubt. Dies erleichtert für viele Patienten die konsequente Medikamenteneinnahme. Auch wird die Inhalation des Steroids begünstigt, das aufgrund fehlender Sofortwirkung ohnehin häufig als wirkungslos betrachtet wird. Neuere Untersuchungen von McCarthy u. Mitarb. zeigen, dass hiermit die Compliance gegenüber einer ICS-Monotherapie um ca. 50\% gesteigert werden kann [5].

In den zurückliegenden Jahren konnte in einer großen Anzahl von Studien die gute klinische Wirksamkeit der freien Kombination von inhalierbaren Steroiden und $\beta$-Mimetika nachgewiesen werden. Es war somit konsequent, ihre Verabreichung in einem
Inhalersystem zu entwickeln. Unterstützt wurde dies durch Ergebnisse aus biochemischen und molekularbiologischen Untersuchungen, die eine synergistische Wirkung beim kombinierten Einsatz der Controller und Relieversubstanz aufzeigten. Durch Besetzung der Kortikoidrezeptoren (CR) und hierdurch vermittelte Stimulation der Kortikoid responsive Elements (CRE) im Zellkern wird vermehrt $\beta$-Adrenozeptor mRNA transkripiert. Dies resultiert in einer Erhöhung der $\beta$-Adrenozeptorpopulation, die an der Zelloberfläche für die $\beta$-Agonistenstimulation zur Verfügung steht. Umgekehrt kann durch $\beta$-Agonisten vermittelte Signaltransduktion CR-Bildung stimuliert und damit die $\beta$-Adrenozeptor-Transkription perpetuiert werden. Steroide können also Anzahl und Ansprechbarkeit von $\beta$-Adrenozeptoren erhöhen, wobei $\beta$-Agonisten auf diesen Prozess selbst günstig Einfluss nehmen können [1].

Die bereits erwähnten ersten klinischen Studien mit der fixen Arzneimittelkombination von ICS und LABA ( = LABACS) zeigten, dass sich sowohl lungenfunktionsanalytische als auch klinische Parameter überadditiv verbesserten. Für dieses überraschende wie reproduzierbare Ergebnis gibt es bisher noch keine plausible Erklärung. Trotzdem eröffnet sich die Möglichkeit, Asthmastadien niedriger dosiert mit ICS behandeln zu können, wenn $\beta$-Agonisten zum regulären Konzept dazu gehören. Allerdings ist die Studienlage mit der fixen LABACS-Kombination derzeit noch nicht sehr ergiebig. Dies trifft insbesondere für das Asthmastadium 2 ( = leichtes, persistierendes Asthma) zu, zumal primär das Interesse auf die Stadien des moderaten und höhergradigen Asthmas fokussierte, bei denen ICS und LABA im therapeutischen Regime verankert sind. 
Das Management der langfristigen Steroidanwendung bei persistierenden Asthmastadien umfasst im Wesentlichen zwei Wege: Einerseits können ICS möglichst niedrig als Monotherapie eingesetzt werden, ergänzt um kurzwirksame $\beta$-Agonisten bei Bedarf und einer Höherdosierung der ICS sowie Ergänzung mit LABA im Fall der Zunahme des Schweregrades [3]. Andererseits ist eine dauerhafte Kombinationsbehandlung mit LABACS möglich und eine auf der Ausnützung von Synergieeffekten zielende minimale LABACS-Menge. Bei letzter Modalität ergibt sich als zusätzlicher Vorteil ein geringerer Bedarf von $\beta$-agonistischer Bedarfsmedikation, zumal bereits regelhaft niedrig dosiert Bronchodilatatoren verabreicht werden.

Welchen Stellenwert kann die fixe Kombination beim schweren Asthma bzw. Asthmaanfall haben? Hier zeigten Rodrigo und Rodrigo, dass die sofortige kombinierte Anwendung von $\beta$-Agonisten und ICS in der Nothilfe im Verlauf der nächsten 2 Stunden eine signifikant bessere Entwicklung der Lungenfunktion erbrachte als dies bei Patienten der Fall war, die nur mit $\beta$-Agonisten behandelt wurden [9]. Auch mussten signifikant weniger kombiniert mit Flunisolid behandelte Patienten im Verlauf stationär aufgenommen werden. Wenngleich diese Studie nicht mit einer fixen, sondern freien Kombination aus Salbutamol und Flunisolid, durchgeführt wurde, so lässt sich in Zusammenschau mit den Ergebnissen aus Asthma-Schweregrad-III-Studien ableiten, dass sich mit der fixen Anwendung von LABACS auch bei schwer erkrankten Patienten ein zusätzlicher Wirkvorteil erwarten lässt. Die überadditive LABACS-Wirkung ist mit Wahrscheinlichkeit durch den permissiven Effekt von Steroiden auf die durch $\beta$-Agonisten vermittelte Signaltransduktion vermittelt. Nachdem eine Reihe von Studien die Hinzunahme von oralen Steroiden beim schweren Asthma klar belegen, kann die vorübergehend hochdosierte, entlang des Obstruktionsgrad titrierte LABACS Anwendung möglicherweise eine Ergänzung oder Änderung des bisherigen therapeutischen Vorgehens darstellen. Hier fehlen allerdings noch weitere repräsentative Studien.

Aktuell gibt es für die Schweregrade 3 und 4 eine solide Datenlage und damit Konsens, dass der Einsatz von LABACS den gegenwärtigen therapeutischen Standard abbildet. Die entscheidende Evidenz wurde mit der FACET-Studie [7] geschaffen. Für Patienten mit mittelschwerem bis schwerem Asthma (Stadium 3) ließ sich hier an über 800 Patienten eindrucksvoll belegen, dass durch die kombinierte Daueranwendung von LABA und niedrig dosiertem ICS eine bessere Lungenfunktion, eine relevant niedrigere Exazerbationsrate und deutlich bessere Symptomscores erzielbar sind, im Vergleich zu den Patienten, die auf eine doppelte ICS-Dosis ohne LABA randomisiert worden sind. Da in dieser Studie die Wirksubstanzen in 2 Inhalern appliziert wurden, ist mit einem noch deutlicheren Wirkvorteil für die Kombination bei fixer Anwendung zu rechnen.

Klinisch konnten Kips u. Mitarb. in einer 12-monatigen Studie die Ergebnisse von Pauwels aus der FACET-Studie bestätigen [4]. Darüber hinaus zeigte sich auch, dass sich die inflammatorische Reaktion der Bronchialschleimhaut beim Asthmatiker nicht verschlechtert, wenn er mit einem niedrigeren ICS-Anteil und dafür kombiniert mit einem $\beta$-Agonisten behandelt wird. Hierfür wurden biochemische und zelluläre Parameter (EG2+-Zellen, ECP, Anzahl der Eosinophilen etc.) der Entzündungsreaktion geprüft.
Sie nahmen zwischen den Patientengruppen keine unterschiedliche Entwicklung, d.h. die Kontrolle der Inflammation blieb erhalten, zusätzlich verbesserte sich die Symptomkontrolle.

Bei der fixen Anwendung der Wirksubstanzen in einem Inhaler scheint sich auch noch der antiasthmatische Wirkeintritt zu beschleunigen. So konnten Zetterström u. Mitarb. in der COMBATStudie eine schnellere Besserung der Lungenfunktion als auch der Asthma Symptomscores in der Gruppe der Patienten verzeichnen, die ICS und LABA in einem Inhaler bekamen, verglichen mit den Patienten, die mit der freien Kombination therapiert wurden [14]. Dies ist bislang nur für Budesonid und Formoterol beschrieben. Ob sich das für Salmeterol und Fluticason in gleicher Weise darstellt, muss sich zeigen.

Nicht unerwartet bringt die mit der LABACS-Anwendung verbundene dauerhafte $\beta$-Agonistenbehandlung die Diskussion um den Verlust an Symptomkontrolle und somit Therapiesicherheit sowie Desensibilisierung wieder in Gang. So wird eine Kaschierung der Entzündungsreaktion ebenso wie die Gefahr der Toleranzentwicklung ins Feld geführt. Für beides gibt es keinen Anhalt. Entsprechend konnte Anne Tattersfield in einer weiteren Analyse der FACET-Daten zeigen, dass sich weder Vorhersagbarkeit noch die Schwere von Exazerbationen bei den einzelnen Therapiegruppen (Bud200, Bud800, Bud200+Form24, Bud800+ Form24) unterschieden, d.h. sich LABA klinisch nicht negativ auswirkte [11]. Eine Verminderung der Symptomkontrolle bei Daueranwendung von $\beta$-Agonisten oder Begünstigung von Exazerbationen zeigte sich in keiner Studie. $\beta$-Agonisten verhindern in der Daueranwendung Exazerbationen und führen nicht zum Gegenteil. Sie verringern die Menge an Bedarfsmedikation und weisen keinerlei klinisch relevante Zeichen von Toleranzentwicklung auf. Auch verbessern LABA zusammen mit ICS die Perzeption bronchialer Obstruktion als auch die bronchiale Hyperreagibilität und erhöhen damit die Symptomkontrolle [2]. Mit den sich nun auf dem Markt befindlichen Kombinationen aus Formoterol und Budesonid sowie Salmeterol und Fluticason bieten sich hervorragende Wirkkombinationen, die mit unterschiedlichen Wirkstärken bedarfsgerecht verordnet und verabreicht werden können.

Unklarheit besteht über das Vorgehen bei Patienten im Asthma Schweregrad 2. Sollen diese nun entgegen der bisheriger Richtlinien fix kombiniert mit LABA behandelt werden, insbesondere wenn sie unter einer ICS-Monotherapie eine adäquate Symptomkontrolle aufweisen, oder nicht? Hier ist die Datenlage noch dünn, aber deswegen nicht weniger interessant.

Grundsätzlich ändert sich vom leichten zum mittelschweren und schweren Asthma die zugrundeliegende pathophysiologische Störung nicht, allenfalls das Ausmaß der Inflammation und ihrer Folgestörungen. Somit sind keine neuen Therapiekonzepte erforderlich, sondern ihre bedarfsgerechte Anpassung an die Schwere der gesundheitlichen Störung. Die Daten, die O'Byrne u. Mitarb. mit der OPTIMA-Studie geschaffen haben, unterstützen dies eindrucksvoll [6]. Hier wurden in einer großen Multizenterstudie zwei Gruppen von leichten Asthmatikern untersucht. Die eine Hälfte der Probanden (Gruppe A) war ohne ICSErhaltungstherapie, so dass sie ein sehr leichtes Asthma aufwiesen, Schweregrad 1 und 2. Die anderen Patienten waren mit 
niedrig dosierten ICS behandelt und bronchodilatatorischer Bedarfsmedikation (Gruppe B) vorbehandelt, d.h. Patienten mit Schweregrad 2.

Durch die Kombinationstherapie konnte bei den leicht erkrankten Patienten (Gruppe A) eine signifikante Verbesserung der Lungenfunktionsparameter erzielt werden. In der Verringerung der Exazerbationsrate $(>30 \%)$ und beschwerdefreien Tagen (15\%) zeigten sich keine zusätzlichen Vorteile einer kombinierten Behandlungsweise mit niedrig dosierten ICS, was den Einsatz einer fixen Kombination aus LABACS nicht unterstützt. Anders bei den mit ICS vorbehandelten, „klassischen“ Schweregrad2-Patienten. Analog zur FACET-Studie wurde auch hier mit der Kombinationstherapie mehr an klinischer Wirkung erzielt als durch eine Verdopplung der ICS-Dosis und zwar sowohl bez. Lungenfunktion als auch hochsignifikant für den primären Endpunkt der Exazerbationsrate.

Auch Taylor u. Mitarb. haben bereits 1998 eine Studie publiziert, in der er eine um fast $50 \%$ verringerte Exazerbationsrate bei stabilen Asthmatikern zeigte, denen ein LABA zu ihrer ICS-Monotherapie zugelegt wurde [12].

In diesem Zusammenhang bedarf die Analyse der Patientendaten von OPTIMA hinsichtlich Exazerbationsraten bei leichten im Vergleich zu moderat bis schwerer erkrankten Asthmatikern besondere Beachtung. Hier zeigt sich, dass die bislang ICS-freien Patienten der OPTIMA-Studie eine annähernd gleiche Exazerbationsrate aufwiesen wie die mittelschweren/schweren Asthmatiker in der FACET-Studie [6]. Das heißt, die zahlenmäßig größte Gruppe der Patienten mit persistierendem Asthma (Stadium 2) weist klinisch erhebliche Zeichen schlechter Symptomkontrolle auf. Das bringen ebenso sowohl die AIR- als auch die AIRE-Studie zum Ausdruck, in denen die befragten Asthmatiker in einem hohen Prozentsatz nicht zuverlässige Krankheitskontrolle aufwiesen [8].

Die Relevanz des antiinflammatorischen Therapieansatzes quer durch die Schweregradklassen wird eindrücklich durch bioptische Untersuchungen an Asthmatikern belegt [13]. Diese wurden an Gesunden, Asthmatikern in Remission und Asthmatiker, die sich in einem aktiven Krankheitszustand befanden, durchgeführt. Als „in Remission befindlich“ wurden dabei Asthmatiker bezeichnet, die 5 Jahre klinisch beschwerdefrei waren. Bei diesen histologischen und immunhistochemischen Untersuchungen zeigte sich, dass sowohl Parameter, die den Entzündungsprozess wie den fortschreitenden Remodellingprozesse an den Atemwegen anzeigen, bei beiden Krankheitsgruppen signifikant erhöht sind. Untereinander waren sie dagegen quantitativ nicht signifikant unterschiedlich, verglichen mit dem Kontrollkollektiv. Diese Entzündungsreaktion drückte sich auch in einer erhöhten bronchialen Reagibilität für AMP aus ebenso wie in einer vermehrten Menge von exhaliertem NO. Diese Daten weisen eindrucksvoll auf einen dauerhaften Entzündungsprozess in den Atemwegen von Asthmatikern hin, verbunden mit Remodellingprozessen. Dies dürfte auch einen relevanten Aspekt darstellen für die hohe Quote an Exazerbationen und variable Symptomkontrolle bei Asthmatikern in niedrigen Krankheitsstadien. In die gleiche Richtung weisen Daten von Sont u. Mitarb., die zei- gen, dass die Reduktion der bronchialen Hyperreaktivität durch angepasste ICS-Dosen relevant die Exazerbationsewahrscheinlichkeit senkt - begleitet von einem Rückgang histologischer Entzündungszeichen [10]. Ob daraus die Empfehlung abgeleitet werden kann, auch leichte Asthmatiker mit sehr niedrigen ICSDosierungen dauerhaft zu therapieren, kann noch nicht endgültig beurteilt werden. Hier könnte aber das Synergiepotenzial von LABACS zur ICS-Dosiseinsparung ideal genutzt werden.

Zusammenfassend gibt es wenig Gründe, Patienten Wirksubstanzen vorzuenthalten, die sowohl eine klinische als auch biochemische Synergie aufweisen, die Adhärenz zu einer etablierten Therapieform fördern, kostengünstiger sind als die Anwendung der Monosubstanzen, die Asthmakontrolle verbessern und hinsichtlich potenzieller Nebenwirkungen Vorteile haben. Somit gibt keine schlüssige Rationale, mit Erreichen eines niedrigen Asthmaschweregrades eine Kehrtwende in therapeutischen Grundsätzen zu vollziehen.

\section{Literatur}

${ }^{1}$ Barnes PJ. Scientific nationale for inhaled combination therapy with long-acting $\beta 2$-agonists and corticosteroids. Eur Respir J 2002; 19: $182-191$

2 Bijl-Hofland ID, Cloostermann SGM, Folgering HThM et al. Inhaled Corticosteroids, combined with long-acting $\beta 2$-agonists, improve the perception of bronchoconstriction in asthma. Am J Respir Crit Care Med 2001; 164: $764-769$

${ }^{3}$ Foresi A, Morelli MC, Catena E. Low-dose budesonide with the addition of an increased dose during exacerbations is effective in longterm asthma control. Chest 2000; 117: 440-446

${ }^{4} \mathrm{Kips} \mathrm{JC,} \mathrm{O'Connor} \mathrm{BJ,} \mathrm{Inman} \mathrm{MD} \mathrm{et} \mathrm{al.} \mathrm{A} \mathrm{long-term} \mathrm{study} \mathrm{of} \mathrm{the} \mathrm{antiin-}$ flammatory effect of low-dose budesonide plus formeterol versus high-dose budesonide in asthma. Am J Respir Crit Care Med 2000; 161: $996-1001$

${ }^{5}$ McCarthy TP, Rice L, Blair CA. Improved compliance with asthma therapy in children treated with salmeterol/fluticasone porprionate combination compared with concurrent salmeterol plus inhaled corticoid or ICS alone. Am J Respir Crit Care Med 2003; 167: A660

${ }^{6}$ O'Byrne PM, Barnes PJ, Rodriguez-Roisin R et al. Low dose inhaled budesonide and formeterol in mild persistent asthma (OPTIMA). Am J Respir Crit Care Med 2001; 164: 1392-1397

7 Pauwels RA, Löfdahl CG, Postma DS et al. Effect of inhaled formeterol and budesonide on exacerbations of asthma (FACET). New Engl J Med 1997; 337: 1405 - 1411

${ }^{8}$ Rabe KF, Vermeire PA, Soriano JB et al. Clinical management of asthma in 1999: the asthma insights and reality in Europe (AIRE) study. Eur Respir J 2000; 16: $802-807$

${ }^{9}$ Rodrigo G, Rodrigo C. Inhaled flunisolide for acute severe asthma. Am J Respir Crit Care Med 1988; 157: 698-703

${ }^{10}$ Sont JK, Willems LNA, Bel EH et al. Clinical control and histopathologic outcome of asthma when using airway hyperresponsiveness as an additional guide to long-term treatment. Am J Respir Crit Care Med 1999; 159: 1043 - 1051

11 Tattersfield AE, Postma DS, Barnes PJ et al. Exacerbations of asthma. Am J Respir Crit Care Med 1999; 160: 594-599

12 Taylor DR, Town GI, Herbison GP et al. Asthma control during longterm treatment with regular inhaled salbutamol and salmeterol. Thorax 1998; 53: 744-752

${ }^{13}$ Toorn LM van den, Overbeck SE, Jongste JC de et al. Airway inflammation is present during clinical remission of atopic asthma. Am J Respir Crit Care Med 2001; 164: 2107 - 2113

${ }^{14}$ Zetterström O, Buhl R, Mellem H et al. Improved asthma control with budesonide/formeterol in a singular inhaler, compared with budesonide alone (COMBAT). Eur Respir J 2001; 18: $262-268$ 